ARTICLE

\title{
Modulated structure determination and ion transport mechanism of oxide-ion conductor $\mathrm{CeNbO}_{4+\delta}$
}

\author{
Jian Li (1) 1,2,7, Fengjuan Pan ${ }^{1,7}$, Shipeng Geng 3,7 , Cong Lin ${ }^{1,4,7}$, Lukas Palatinus (10) ${ }^{5}$, Mathieu Allix (i) ${ }^{6}$, \\ Xiaojun Kuang (iD ${ }^{3 凶}$, Jianhua $\operatorname{Lin}^{1} \&$ Junliang Sun (1) ${ }^{1,2 \otimes}$
}

$\mathrm{CeNbO}_{4+\delta}$, a family of oxygen hyperstoichiometry materials with varying oxygen content $\left(\mathrm{CeNbO}_{4}, \mathrm{CeNbO}_{4.08}, \mathrm{CeNbO}_{4.25}, \mathrm{CeNbO}_{4.33}\right)$ that shows mixed electronic and oxide ionic conduction, has been known for four decades. However, the oxide ionic transport mechanism has remained unclear due to the unknown atomic structures of $\mathrm{CeNbO}_{4.08}$ and $\mathrm{CeNbO}_{4.33}$. Here, we report the complex $(3+1) \mathrm{D}$ incommensurately modulated structure of $\mathrm{CeNbO}_{4.08}$, and the supercell structure of $\mathrm{CeNbO}_{4.33}$ from single nanocrystals by using a threedimensional electron diffraction technique. Two oxide ion migration events are identified in $\mathrm{CeNbO}_{4.08}$ and $\mathrm{CeNbO}_{4.25}$ by molecular dynamics simulations, which was a synergiccooperation knock-on mechanism involving continuous breaking and reformation of $\mathrm{Nb}_{2} \mathrm{O}_{9}$ units. However, the excess oxygen in $\mathrm{CeNbO}_{4.33}$ hardly migrates because of the high concentration and the ordered distribution of the excess oxide ions. The relationship between the structure and oxide ion migration for the whole series of $\mathrm{CeNbO}_{4+\delta}$ compounds elucidated here provides a direction for the performance optimization of these compounds.

\footnotetext{
${ }^{1}$ College of Chemistry and Molecular Engineering, Peking University, 100871 Beijing, P. R. China. ${ }^{2}$ Department of Materials and Environmental Chemistry, Stockholm University, 10691 Stockholm, Sweden. ${ }^{3}$ Guangxi Key Laboratory of Optical and Electronic Materials and Devices, College of Materials Science and Engineering, Guilin University of Technology, 541004 Guilin, P. R. China. ${ }^{4}$ School of Advanced Materials, Shenzhen Graduate School, Peking University, 518055 Shenzhen, P. R. China. ${ }^{5}$ Institute of Physics, Academy of Sciences of the Czech Republic, v.v.i., Na Slovance 2, 18221 Prague, Czech Republic. ${ }^{6}$ CNRS, CEMHTI UPR3079, Univ. Orléans, 45071 Orléans, France. ${ }^{7}$ These authors contributed equally: Jian Li, Fengjuan Pan, Shipeng Geng, Cong Lin.

凶email: kuangxj@glut.edu.cn; junliang.sun@pku.edu.cn
} 
M aterials with oxygen hyperstoichiometry have excellent electronic, magnetic, and oxygen storage properties and can be used in a wide variety of applications ${ }^{1-5}$. For example, $\mathrm{La}_{2} \mathrm{CuO}_{4+\delta}$ forms two phases depending on the oxygen content ${ }^{6}$ : the phase with $\delta=0$ is semiconducting, while the other has a wide range of values $(0.03<\delta<0.18)$ and it becomes superconducting for $\delta=0.08$ with $T_{\mathrm{c}} \sim 38 \mathrm{~K}$. The discovery of the series of 114 cobaltites $\left((\mathrm{Ln}, \mathrm{Ca})_{1} \mathrm{BaCo}_{4} \mathrm{O}_{7}\right)$ revealed the existence of closely related structures with various crystallographic symmetries and the possibility of oxygen nonstoichiometry in the range " $\mathrm{O}_{7}$ "- " $\mathrm{O}_{8.5}$ " in those systems, which opened up a new field for the investigation of strongly correlated electron systems. This change of oxygen stoichiometry, which induces the variation of the $\mathrm{Co}^{2+}: \mathrm{Co}^{3+}$ ratio in the system, is expected to influence the physical properties of these compounds considerably. This is the case of the oxygen-rich "114" cobaltites $\mathrm{YBaCo}_{4} \mathrm{O}_{8}$ and $\mathrm{YbBa}$ $\mathrm{Co}_{4} \mathrm{O}_{7.2}$, which were shown to be magnetically frustrated rather than magnetically ordered at low temperatures ${ }^{7,8}$. Therefore, oxygen hyperstoichiometry materials with useful functionalities are attractive subjects of research for chemists, physicists, or materials scientists.

In recent years, materials with oxygen hyperstoichiometry received great attention in the field of solid oxide fuel cells because of the low activation energy $\left(E_{\mathrm{a}}\right)$ of interstitial ion migration. Well known and studied examples are apatites $\mathrm{La}_{10}$ ${ }_{\delta}\left(\mathrm{MO}_{4}\right)_{6} \mathrm{O}_{3-1.5 \delta}(\mathrm{M}=\mathrm{Si}, \mathrm{Ge})^{9}$, melilite $\mathrm{La}_{1+\delta} \mathrm{Sr}_{1-\delta} \mathrm{Ga}_{3} \mathrm{O}_{7+0.5 \delta}{ }^{10}$, layered perovskites $\mathrm{La}_{2} \mathrm{NiO}_{4+\delta}{ }^{11}$, fluorite $\mathrm{UO}_{2+\delta} \delta^{12}$, or scheelite $\mathrm{La}_{0.2} \mathrm{~Pb}_{0.8} \mathrm{WO}_{4+\delta}{ }^{13}, \mathrm{Bi}_{1-\delta} \mathrm{Sr}_{\delta} \mathrm{VO}_{4-0.5 \delta}{ }^{14}$, and also the subject of this study, $\mathrm{CeNbO}_{4+\delta}$. $\mathrm{CeNbO}_{4+\delta}$ is a mixed ionic and p-type electronic conductor with fast oxygen ion diffusion at moderate temperatures (total conductivity up to $0.030 \mathrm{~S} \mathrm{~cm}^{-1}$ at $850{ }^{\circ} \mathrm{C}$; ion transference number up to 0.4 ; diffusion coefficient up to $8.3 \times 10^{-8} \mathrm{~cm}^{2} \mathrm{~s}^{-1}$ ), making it a promising material for applications in energy generation and storage devices ${ }^{15-23}$. $\mathrm{CeNbO}_{4+\delta}$ was first reported by Cava et al. ${ }^{15}$ in 1970s. It has been identified as a family of compounds with variable oxygen contents, with distinct phases $\mathrm{CeNbO}_{4}, \mathrm{CeNbO}_{4.08}, \mathrm{CeNbO}_{4.25}$, and $\mathrm{CeNbO}_{4.33}$. Although Thompson et al. ${ }^{16}$ successfully indexed the unit cell of these four compounds by selected area electron diffraction (SAED) in 1999, no progress on the structure solution of these phases was made until in 2016 Pramana et al. ${ }^{17}$ solved the structure of $\mathrm{CeNbO}_{4.25}$ by single-crystal X-ray diffraction (SCXRD) and revealed by molecular dynamics (MD) simulations that the fast ion migration occurs within planes of the neighboring $\mathrm{NbO}_{n}$ polyhedra. However, the atomic structures of $\mathrm{CeNbO}_{4.08}$ and $\mathrm{CeNbO}_{4.33}$ remained unknown, which hindered the full understanding of the oxide ion conduction behavior for the whole system of $\mathrm{CeNbO}_{4+\delta}$.

In order to better understand the oxygen transport mechanism and to optimize its performance, atomic structures of $\mathrm{CeNbO}_{4.08}$ and $\mathrm{CeNbO}_{4.33}$ need to be understood. However, for $\mathrm{CeNbO}_{4.08}$ and $\mathrm{CeNbO}_{4.33}$, it was very difficult to grow large single crystal due to their specific syntheses. Furthermore, $\mathrm{CeNbO}_{4.08}$ appeared to be a $(3+2)$-dimensional incommensurately modulated structure with monoclinic symmetry and $\mathrm{CeNbO}_{4.33}$ a commensurately modulated structure with triclinic symmetry according to SAED ${ }^{16}$. The large unit cell parameters and complex diffraction patterns make it very difficult to determine their atomic structures by conventional powder X-ray diffraction (PXRD). Fortunately, nanocrystals and microcrystals can be treated as single crystals in electron microscopy. The recently developed 3D ED technique, continuous rotation $\mathrm{ED}^{24-27}$, can use single nanocrystals to obtain single-crystal diffraction data that can be used for structure determination by utilizing the software developed for SCXRD (ShelxT ${ }^{28}$, Superflip ${ }^{29,30}$ ).
In this study, we determine the incommensurately modulated structure of $\mathrm{CeNbO}_{4.08}$ and the superstructure of $\mathrm{CeNbO}_{4.33}$ by combining 3D ED, synchrotron X-ray powder diffraction (SPD), and neutron powder diffraction (NPD). Using the same methods, the superstructure of $\mathrm{CeNbO}_{4.25}$ was also re-determined. The structure models are sufficiently accurate to reveal the interstitial oxygen sites and allow to elucidate how the extra oxygen atoms change the structural connectivity. Combining the structural information with MD simulations, we describe the oxide ion migration mechanisms in $\mathrm{CeNbO}_{4+\delta}$. The relationship between the structure and oxide ion migration for the whole series of $\mathrm{CeNbO}_{4+\delta}$ compounds here provides means to optimize the performance of these compounds and to develop better oxygen hyperstoichiometric materials for a wide variety of applications.

\section{Results}

A series of phases of $\mathrm{CeNbO}_{4+\delta}$ was obtained from $\mathrm{CeO}_{2}$ and $\mathrm{Nb}_{2} \mathrm{O}_{5}$ by the solid-state synthetic route in Supplementary Fig. 1 . The most striking chemical feature of the studied cerium niobate phases is that they are progressively oxidized from one structure to another under relatively mild conditions. The oxygen contents of the phases were analyzed by thermogravimetric analysis (TGA), which yields formulae $\mathrm{CeNbO}_{4.11(2)}, \mathrm{CeNbO}_{4.24(2)}$, and $\mathrm{CeNbO}_{4.33(1)}$ (Supplementary Fig. 2). Figure 1 shows the $[010]_{\mathrm{p}}$ ( $\mathrm{p}$ denotes parent material $\mathrm{CeNbO}_{4}$ ) zone axis SAED patterns of the $\mathrm{CeNbO}_{4+\delta}(\delta=0,0.08,0.25,0.33)$ phases. In all the four SAED patterns, the strong reflections have the same pseudotetragonal symmetry as that of the parent structure. However, for $\mathrm{CeNbO}_{4.08}, \mathrm{CeNbO}_{4.25}$, and $\mathrm{CeNbO}_{4.33}$, many additional reflections can be observed.

Incommensurately modulated structure of $\mathrm{CeNbO}_{4.08}$. In previous transmission electron microscopic study, $\mathrm{CeNbO}_{4.08}$ was identified as a monoclinic $(3+2) \mathrm{D}$ incommensurately modulated phase with average unit cell parameters close to the parent $\mathrm{CeNbO}_{4}$ and modulation vectors $\mathrm{q} 1 \sim(0.138,0,0.344), \mathrm{q} 2 \sim$ $(0.345,0,-0.138)$ and centering $I=(1 / 2,1 / 2,1 / 2,0,0)$ (Supplementary Fig. 3a ${ }^{16}$. In the present study, analysis of the $[010]_{p}$
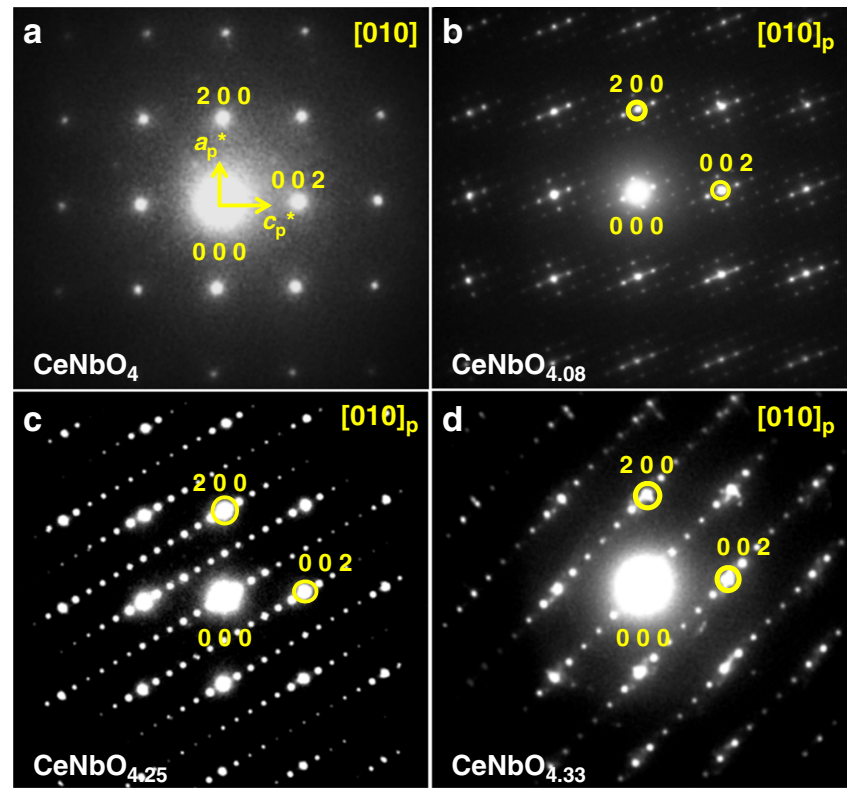

Fig. 1 [010] $]_{\mathbf{p}}$ zone axis SAED of $\mathbf{C e N b O}_{\mathbf{4}+\boldsymbol{\delta}}$ a $\mathrm{CeNbO}_{4.0}$. b $\mathrm{CeNbO}_{4.08}$. c $\mathrm{CeNbO}_{4.25}$. d $\mathrm{CeNbO}_{4.33}$. The strong reflections in $\mathbf{b}$-d have the same pseudo-tetragonal symmetry as the reflections of the parent structure in $\mathbf{a}$ ( $p$ denotes parent material $\mathrm{CeNbO}_{4}$ ). 


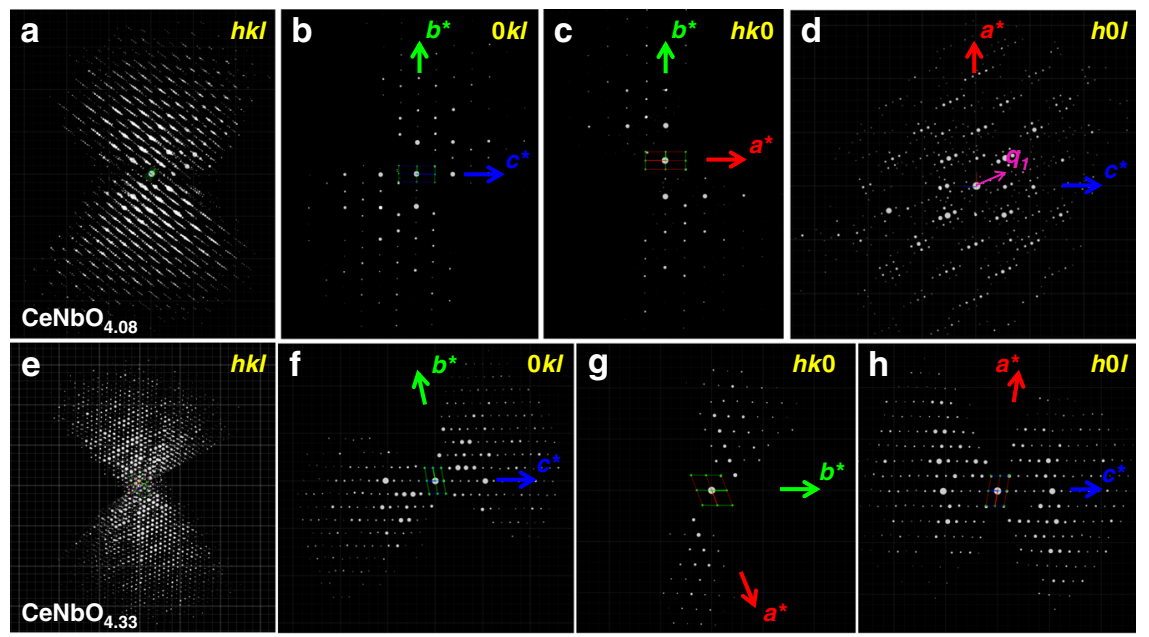

Fig. 2 3D ED data of $\mathbf{C e N b O}_{\mathbf{4 . 0 8}}$ and $\mathbf{C e N b O}_{4.33} .3 \mathrm{D}$ reciprocal lattice reconstructed from the 3D ED data of $\mathrm{CeNbO}_{4.08}(\mathbf{a})$ and $\mathrm{CeNbO} 4.33(\mathbf{e})$. b $(0 \mathrm{kl})$, c $(h k 0)$, and $\mathbf{d}(h O l)$ slices extracted from the reconstructed reciprocal lattice of $\mathrm{CeNbO}_{4.08}$, reflection conditions: $0 \mathrm{klm}: k+I=2 n . h k 0 \mathrm{~m}: h+k=2 n, h O l m:$ $h+m=2 n, I+m=2 n, h 00 m: h+m=2 n, 0 k 00: k=2 n, 00 I m: I+m=2 n . \mathbf{f}(0 k l), \mathbf{g}(h k 0)$, and $\mathbf{h}(h O l)$ slices cut from the reconstructed reciprocal lattice of $\mathrm{CeNbO}_{4.33}$, reflection conditions: no special systematic reflections appear forbidden.
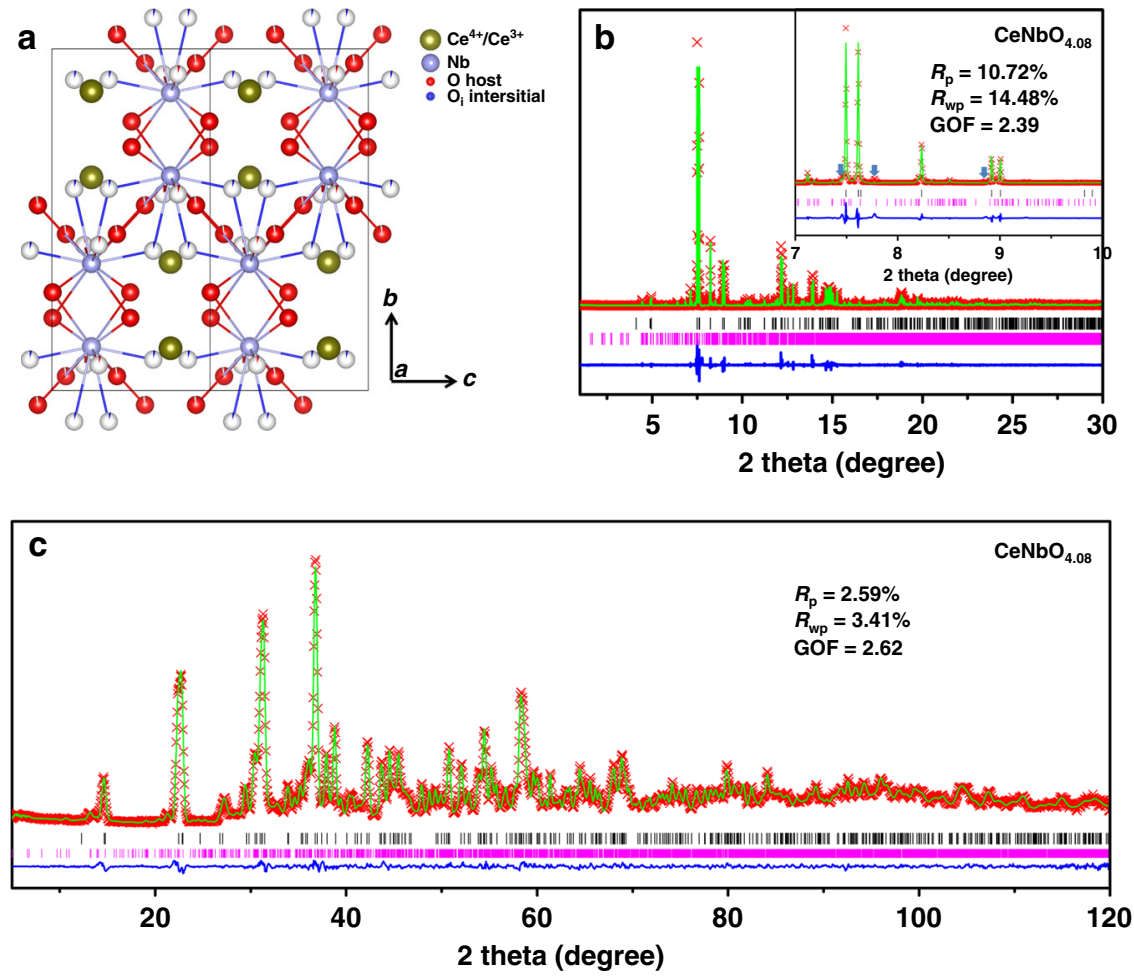

Fig. 3 Structure characterization and analysis of $\mathbf{C e N b O}_{\mathbf{4 . 0 8}}$. a Average structure of the $(3+1) \mathrm{D}$ incommensurately modulated structure of $\mathrm{CeNbO}$.08 along the $[100]_{p}$ direction. The interstitial $\mathrm{O} 3$ (blue atom) is located between Ce cations within the Ce cationic chain. $\mathbf{b}$ Final Rietveld refinement against the SPD data for $\mathrm{CeNbO}_{4.08}$ with $(3+1) \mathrm{D}$ superspace. Note: the unexplained peaks at 7.46, 7.77, and 8.86 degree (marked at inset pattern) belong to the impurity phase of $\mathrm{CeNbO}_{4.25}$. c Final Rietveld refinement against the NPD data for $\mathrm{CeNbO}_{4.08}$ with $(3+1) \mathrm{D}$ superspace. (Red multi ( $x$ ) symbol: observed profile, green curve: simulated profile, blue curve: difference profile, black Bragg line: main reflections, pink Bragg line: satellite reflections).

zone axis SAED of $\mathrm{CeNbO}_{4.08}$ indicated that the structure can be described as monoclinic $(3+1) \mathrm{D}$ incommensurately modulated with a single modulation vector $q=0.069 a^{*}+0.175 c^{*}$ and a nonstandard centering $X=(1 / 2,1 / 2,1 / 2,1 / 2)$. However, satellites up to order 5 or even 7 are needed to describe the whole pattern (Supplementary Fig. 3b). The qualities of the LeBail fit of SPD are similar for the 2D modulation and $1 \mathrm{D}$ modulation, which further confirmed that the $1 \mathrm{D}$ modulation is the correct description (Supplementary Fig. 4). It is extremely unlikely that a single modulation vector would be able to describe two independent modulation vectors just by coincidence with such accuracy. Satellites up to order 7 in the synchrotron powder data are visible. However, the number of satellites is very large and the sensitivity of the SPD pattern to oxygen positions is relatively low, making it extremely challenging to solve the $(3+1) \mathrm{D}$ incommensurately modulated structure of $\mathrm{CeNbO}_{4.08}$ ab initio from the SPD pattern. Therefore, the 3D ED technique was applied to solve its incommensurately modulated structure. 
a
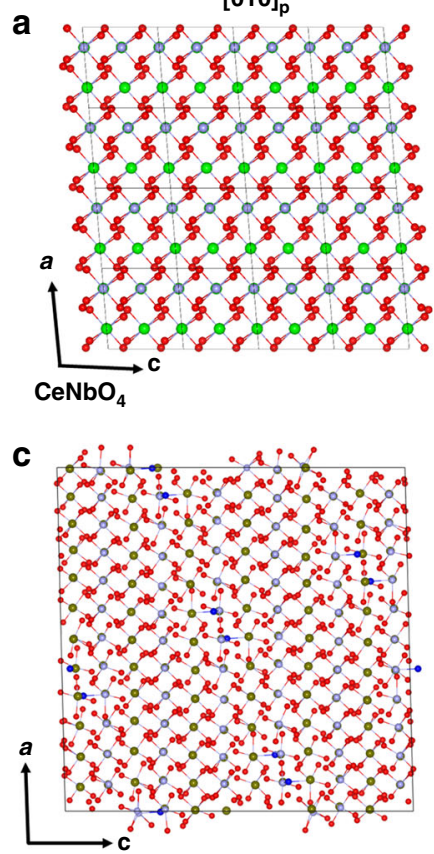

$\mathrm{CeNbO}_{4.08}$

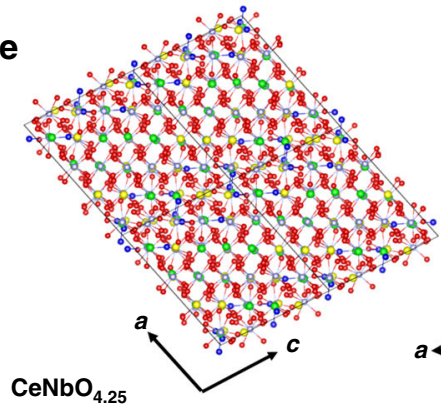

b $[100]_{p}$
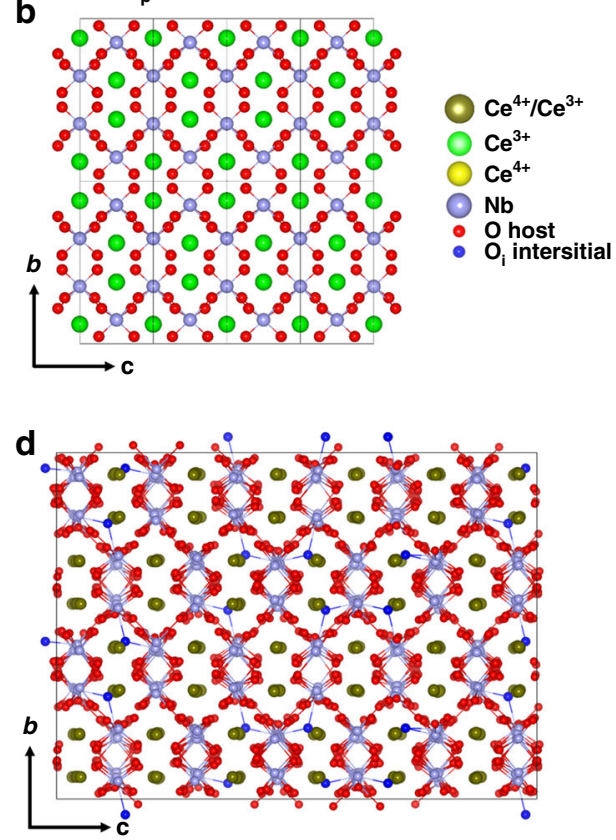
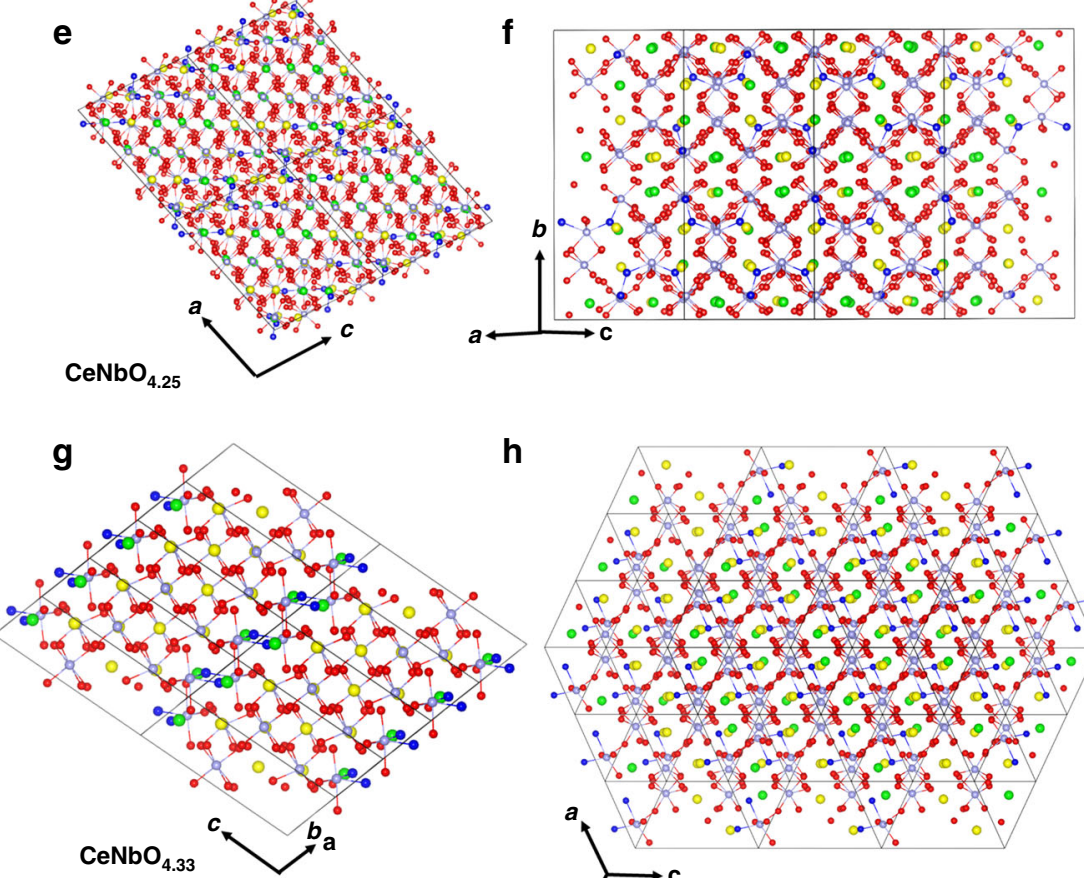

h

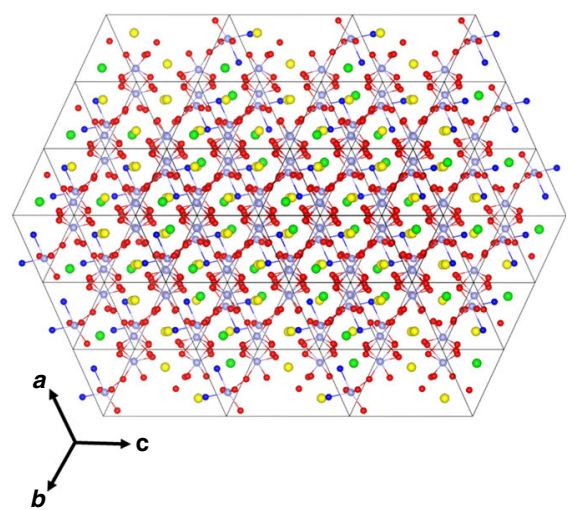

Fig. 4 Structure model of $\mathbf{C e N b O}_{\mathbf{4}+\delta}$. $\mathrm{CeNbO}_{4}(\mathbf{a}, \mathbf{b})$, oxidized $\mathrm{CeNbO}_{4.08}(\mathbf{c}, \mathbf{d}), \mathrm{CeNbO}_{4.25}(\mathbf{e}, \mathbf{f})$, and $\mathrm{CeNbO}_{4.33}(\mathbf{g}$, $\mathbf{h})$ projected along the principal axes of $[010]_{p}$ and $[100]_{p}$. Displacement of oxygen from the original position in the parent structure is observed. Note that $\mathbf{c}$ and $\mathbf{d}$ were $6 a \times 2 b \times 6 c$ approximant superstructure in $(3+1) \mathrm{D}$ incommensurately modulated model along [010]p and [100]p.

Due to the limitation of processing continuous mode 3D ED data with $(3+1) D$ superspace, we processed the $3 \mathrm{D}$ ED data with approximate supercell. The $3 \mathrm{D}$ reciprocal lattice reconstructed from the 3D ED data of $\mathrm{CeNbO}_{4.08}$ are shown in Fig. 2a-d. Reciprocal lattice along the $[010]_{\mathrm{p}}$ direction can be cut from the reconstructed 3D reciprocal lattice (Supplementary Fig. 6b), in which the strong reflections show the similar pseudo-tetragonal symmetry as the $[010]_{\mathrm{p}}$ zone axis SAED of $\mathrm{CeNbO}_{4}$. Then we transformed the processed 3D ED data (hkl file based on the approximate supercell) to $(3+1) \mathrm{D}$ superspace. Reflection conditions indicated the superspace group of $X 2 / c(\alpha 0 \gamma) 0 s$ and satellites up to order 7 were required. The determination of the modulation functions turned out to be extremely difficult due to their complexity and large number of contributing harmonic waves. Finally, the $(3+1) \mathrm{D}$ model was constructed from the supercell structure. The structure contains one additional oxygen site (O3) compared with the parent structure of $\mathrm{CeNbO}_{4}$ (Fig. 3a, see Supplementary Information for details on the localization of 

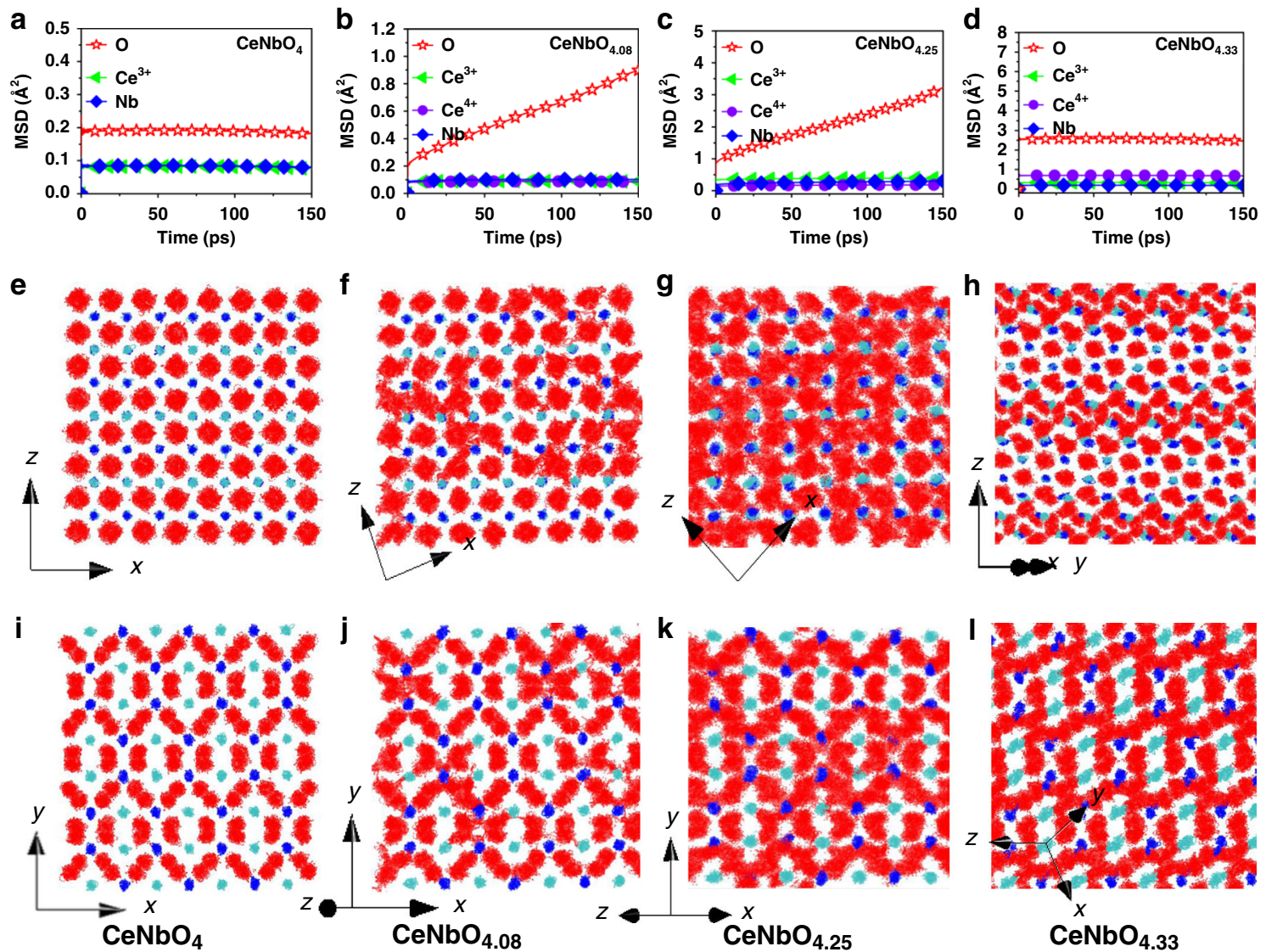

k
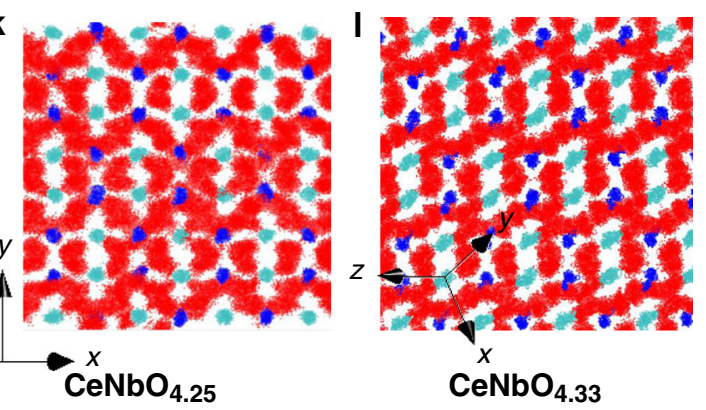

Fig. 5 Molecular dynamics simulations study of $\mathbf{C e N b O}_{4+\delta}$. Calculated MSD values of $\mathrm{Ce}^{3+/ 4+}, \mathrm{Nb}$, and $\mathrm{O}$ atoms as a function of simulation time for $\mathrm{CeNbO}_{4}(\mathbf{a}), \mathrm{CeNbO}_{4.08}(\mathbf{b}), \mathrm{CeNbO}_{4.25}(\mathbf{c})$, and $\mathrm{CeNbO}_{4.33}$ (d) from the $\mathrm{MD}$ simulation at $1200^{\circ} \mathrm{C}$. Trajectory scatter plots for $\mathrm{CeNbO}_{4}(\mathbf{e}, \mathbf{i}), \mathrm{CeNbO}_{4.08}$ $(\mathbf{f}, \mathbf{j}), \mathrm{CeNbO}_{4.25}(\mathbf{g}, \mathbf{k})$, and $\mathrm{CeNbO}_{4.33}(\mathbf{h}, \mathbf{I})$ at $1200^{\circ} \mathrm{C}$ where cyan, blue, and red dots represent $\mathrm{Ce}^{3+/ 4+}, \mathrm{Nb}^{5+}$, and $\mathrm{O}^{2-}$ atoms, respectively.

this additional atoms). Detailed analysis of the interatomic distances and difference potential maps showed that the atom $\mathrm{O} 1$ has two positions-one is the main atomic domain while the other is occupied only when O1 coexists with O3. The modulation structure model was first refined against 3D ED with modulation functions up to order 7 and modulation of ADP parameters up to order 3 for $\mathrm{Ce}$ and 2 for $\mathrm{Nb}$. The final modulated structure model of $\mathrm{CeNbO}_{4.08}$ was obtained by the Rietveld refinement of combined SPD data (Fig. 3b) and NPD data (Fig. 3c). Modulation functions up to order 7 were used together with isotropic atom displacement parameters. The model resulted in chemically reasonable bond distances (Supplementary Fig. 9) and modulation functions similar to the model obtained against 3D ED data (Supplementary Figs. 10 and 11).

Supercell structure of $\mathrm{CeNbO}_{4.33}$. From the $[010]_{\mathrm{p}}$ zone axis SAED, the satellites in $\mathrm{CeNbO}_{4.25}$ and $\mathrm{CeNbO}_{4.33}$ can be indexed with a single vector $q=1 / 12[204]_{\mathrm{p}}$ and $1 / 3[101]_{\mathrm{p}}$, respectively. Thus the commensurately modulated structure of $\mathrm{CeNbO}_{4.25}$ and $\mathrm{CeNbO}_{4.33}$ can be described by supercell models. Again, 3D ED data were collected on $\mathrm{CeNbO}_{4.33}$ (Fig. 2e-h) and $\mathrm{CeNbO}_{4.25}$ (Supplementary Fig. 5e-h). The reflection conditions derived from the $3 \mathrm{D} \mathrm{ED}$ datasets indicated that the possible space group of $\mathrm{CeNbO}_{4.33}$ is $P \overline{1}$. All the crystallographically unique Ce and $\mathrm{Nb}$ atoms as well as a part of oxygen atoms in the structure were located directly by using the software $S H E L X T^{28}$. The supercell structure model of $\mathrm{CeNbO}_{4.25}$ could also be obtained with 3D ED data. The final supercell structure model for $\mathrm{CeNbO}_{4.33}$ and $\mathrm{CeNbO}_{4.25}$ with interstitial oxygen sites were obtained by the
Rietveld refinement of combined SPD and NPD data (Supplementary Figs. 12-15).

Identification of the interstitial sites $\mathrm{O}_{\mathrm{i}}$ in the structure models. Comparing the three oxide supercell structures with the parent structure (Fig. 4 and Supplementary Fig. 16), the additional oxygen atoms incorporated into the $\mathrm{CeNbO}_{4}$ host lattice have relaxed the original oxide ion positions, which resulted in three different incommensurately or commensurately modulated structures. Figure 4 shows the structures of the parent $\mathrm{CeNbO}_{4}$ and the structures of $\mathrm{CeNbO}_{4.08}, \mathrm{CeNbO}_{4.25}$, and $\mathrm{CeNbO}_{4.33}$ projected along the principal axes of $[010]_{\mathrm{p}}$ and $[100]_{\mathrm{p}}$. Significant displacement of oxygen atoms from the original positions in the parent structure is observed and additional oxygen sites appear in the cationic layers, close to the interstitial position $\mathrm{O} 3$ identified in the average structure of $\mathrm{CeNbO}_{4.08}$ (Fig. 3a).

In the oxidized supercell structures, the excess oxygen atoms are located at one $((3+1) \mathrm{D}$ incommensurately modulated model of $\left.\mathrm{CeNbO}_{4.08}\right)$, three $\left(\mathrm{CeNbO}_{4.25}\right)$, and one $\left(\mathrm{CeNbO}_{4.33}\right)$ fully occupied general Wyckoff sites, respectively. The interstitial sites are the blue atoms in Fig. 4 (O3 in $\mathrm{CeNbO}_{4.08} 6 a \times 2 b \times 6 c$ approximant superstructure structure, O4, O11, and $\mathrm{O} 41$ in $\mathrm{CeNbO}_{4.25}$ and $\mathrm{O} 13$ in $\mathrm{CeNbO}_{4.33}$ ). Bond valence sum (Supplementary Tables 2 and 3 and Supplementary Fig. 17) analysis shows that some of the Ce positions are $\mathrm{Ce}^{4+}$ sites. The change of the oxidation state of $\mathrm{Ce}$ is responsible for the shorter $\mathrm{Ce}-\mathrm{O}$ bond lengths and allows that excess oxygen is incorporated into the host lattice at the interstitial sites between Ce cations (Fig. 4c-h). In $\mathrm{CeNbO}_{4.33}$, the amount of additional oxygen is so much that part of the oxygen atoms are pushed away from the original 

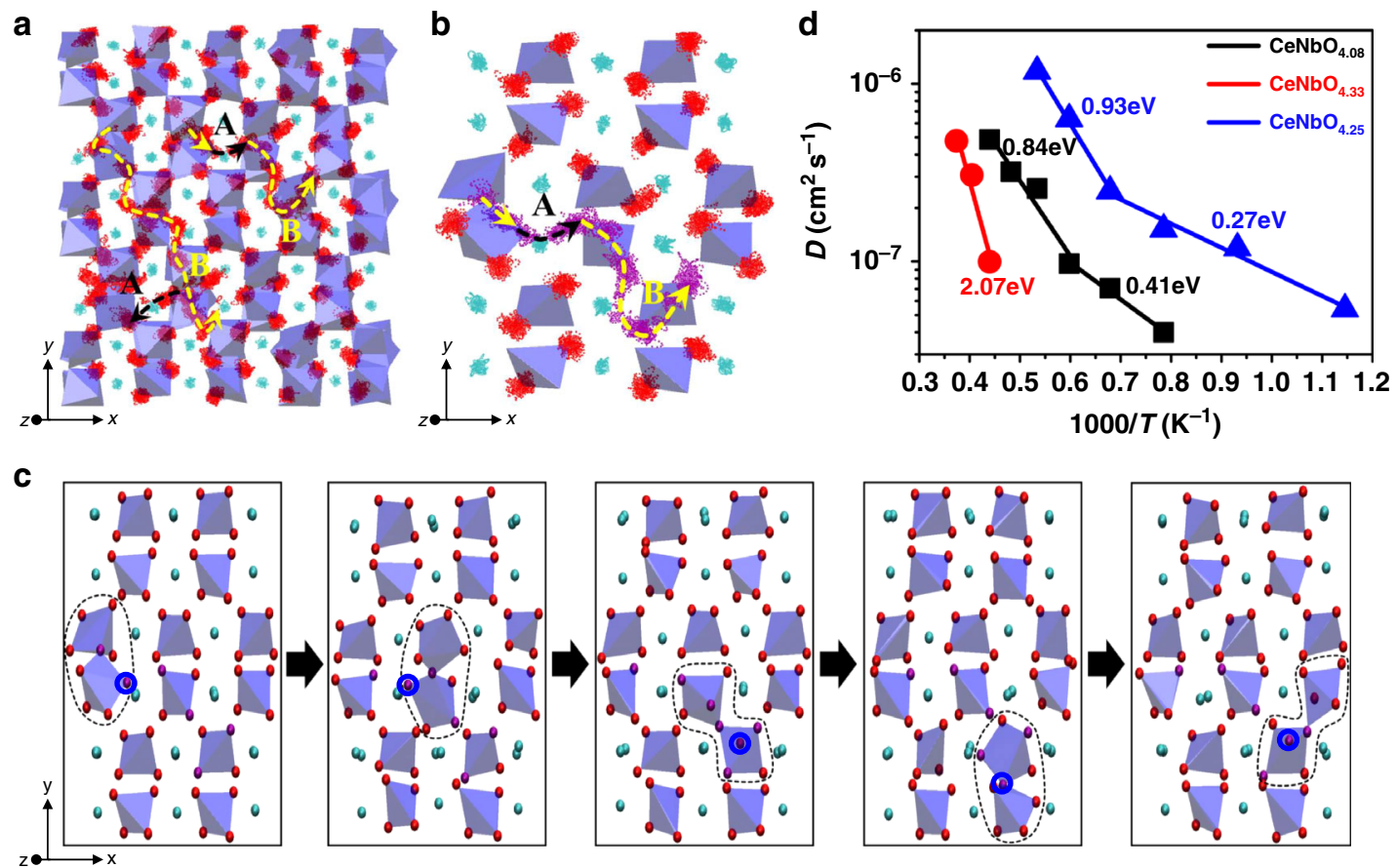

Fig. 6 The oxide ionic transport mechanism of $\mathbf{C e N b O}_{\mathbf{4}+\delta} \mathbf{a}, \mathbf{b}$ Trajectory scatter plots of $\mathrm{Ce}$ and $\mathrm{O}$ atoms showing the migration paths $\mathrm{A}$ (black dash dot line) and $\mathrm{B}$ (yellow dash dot line) with the $\mathrm{NbO}_{n}$ polyhedral network embedded in, and c snapshots of long-range oxide ion (marked with the blue circle) migration involving the breaking and reforming of the $\mathrm{Nb}_{2} \mathrm{O}_{9}$ units (highlighted by dashed lines) in $\mathrm{CeNbO}_{4.08}$ from the $\mathrm{MD}$ simulations at $1200{ }^{\circ} \mathrm{C}$. In order to illustrate the $\mathrm{Nb}_{2} \mathrm{O}_{9}$ breaking and reforming mechanism clearly, in $\mathbf{b}$, $\mathbf{c}$ the weak Nb-O bonds $>2.2 \AA$ are not included in the $\mathrm{NbO}_{n}$ polyhedral units. d Arrhenius plot of the oxygen diffusion coefficient calculated using the MSD values of oxygen atoms for $\mathrm{CeNbO}_{4+\delta}(\delta=0.08,0.25,0.33)$. The activation energies are labeled.

normal site into an interstitial site $(\mathrm{O} 11)$ due to the relaxation of the structure. The interstitial site oxygens $(\mathrm{O} 11, \mathrm{O} 13)$ between $\mathrm{Ce}$ cations are occupied in an ordered manner along the $a$ and $c$ axes in $\mathrm{CeNbO}_{4.33}$ (Supplementary Fig. 18). The ordered interstitial oxygen (O13) and the relaxation of the other oxygen site (O11) generate a zigzag-shaped $\left[\mathrm{Nb}_{6} \mathrm{O}_{26}\right]_{\infty}$ infinite chain comprising edge-sharing $\left[\mathrm{NbO}_{6}\right]_{4}$ units bridged by edge-sharing $\left[\mathrm{NbO}_{6}\right]_{2}$ units through vertex (Supplementary Fig. 18a).

Oxide ion migration. MD simulations based on interatomic potential method were performed to elucidate the dependence of oxide ion migration on the variation of oxygen hyperstoichiometry and structure in $\mathrm{CeNbO}_{4+\delta}$ compounds. The mean square displacement (MSD) values (Fig. 5a-d and Supplementary Fig. 19) and scatter plots (Fig. 5e-l and Supplementary Fig. 20) indicate that all atoms in the parent $\mathrm{CeNbO}_{4}$ show only lattice vibration without long-range migration as expected. With inclusion of the excess oxygen into the host lattice of $\mathrm{CeNbO}_{4}$, the oxide ions become mobile in $\mathrm{CeNbO}_{4.08}$ and $\mathrm{CeNbO}_{4.25}$ but hardly migrate in $\mathrm{CeNbO}_{4.33}$. The $\mathrm{MD}$ simulations show that $\mathrm{CeNbO}_{4.08}$ and $\mathrm{CeNbO}_{4.25}$ phases have similar oxide ion migration pathways.

Two kinds of oxide ion migration events contributing to the long-range migration can be identified in the MD simulations of $\mathrm{CeNbO}_{4.08}$ (Fig. 6a, b). The first one is oxide ion migration between $\mathrm{NbO}_{n}$ polyhedra isolated by the Ce cationic chain by $\sim 5.0-5.4 \AA$ (labeled as path A). In this migration event, the oxide ion leaves one $\mathrm{NbO}_{n}$ polyhedron and passes through the interstitial sites (referred to as $\mathrm{O}_{\mathrm{i}}$ ) within the chain of Ce cations along the $a$ or $c$ axis of the parent $\mathrm{CeNbO}_{4}$ structure to subsequently enter into the coordination environment of another $\mathrm{NbO}_{n}$ polyhedron located at the other side of the $\mathrm{Ce}$ chain through a knock-on process. The second type of migration is the oxide ion migration between the neighboring $\mathrm{NbO}_{n}$ polyhedra separated by $\sim 3.6-4.2 \AA$ (labeled as path $\mathrm{B}$ ) through a chain knock-on process via the $\mathrm{NbO}_{n}$ polyhedral rotation and deformation. This migration event also frequently involves the interstitial sites $\mathrm{O}_{\mathrm{i}}$ (Fig. 6a, b). MD simulations indicate that the migration along path $\mathrm{A}$ is much less frequent than that along path $\mathrm{B}$, which is consistent with the larger separation between the $\mathrm{NbO}_{n}$ polyhedral units involved in path $\mathrm{A}$ than that in path $\mathrm{B}$.

As $\mathrm{Nb}$ atoms are usually found in 6 or 4 coordinate environments and the $\mathrm{Nb}-\mathrm{O}$ bond lengths normally vary within 1.8-2.2 $\AA$, the $\mathrm{Nb}-\mathrm{O}$ bonds $>2.2 \AA$ were not included in the $\mathrm{NbO}_{n}$ polyhedral units during a close examination of oxide ion migration, which simplifies the description of the polyhedral units and assists understanding the complex migration process. This representation leads to isolated tetrahedral $\mathrm{NbO}_{4}$ units and corner-sharing $\mathrm{Nb}_{2} \mathrm{O}_{9}$ units most of the time during simulation. The oxide ions were found to move between the polyhedral units in the long-range migration routes composed of paths $\mathrm{A}$ and $\mathrm{B}$ mainly through the synergic-cooperation mechanism involving the continuous breaking and reformation of the $\mathrm{Nb}_{2} \mathrm{O}_{9}$ units assisted by rotation and deformation of the $\mathrm{NbO}_{n}$ polyhedra and a knock-on process between the oxygen atoms (Fig. 6b, c and Supplementary Video 1). Therefore, the interstitial sites within the Ce cationic chain and $\mathrm{NbO}_{n}$ polyhedra define more like 3D pathways for the oxide ion migration in $\mathrm{CeNbO}_{4.08}$, which is consistent with the fact that inclusion of the extra oxygen atoms disturbs anisotropically the polyhedral linkage in $\mathrm{CeNbO}_{4}$.

The same oxide ion migration pathways are found also in $\mathrm{CeNbO}_{4.25}$ phase in our study. Oxide migration in $\mathrm{CeNbO}_{4.25}$ was analyzed also by Pramana et al. ${ }^{17}$. They identified anisotropic oxide ion diffusion paths within the linked $\mathrm{NbO}_{6}$ layers and pointed out that migration between the adjacent layers also took 
place when the migration paths along the linked $\mathrm{NbO}_{6}$ layers were blocked. The migration routes proposed by Pramana et al. ${ }^{17}$ are similar to those composed of paths B only identified in our study. However, the work of Pramana et al. ${ }^{17}$ did not identify the interstitial sites and as a consequence did not recognize migration paths going through the interstitial sites and the related dynamic process of the adaptation of the polyhedral units to the oxide ion migration. The proper description of the interstitial sites turns out to be crucial for the correct description of the full 3D long-range migration and also for the elucidation of the knock-on dynamic process of breaking and reformation of the $\mathrm{Nb}_{2} \mathrm{O}_{9}$ units during the oxide ion migration.

The calculated oxide diffusion coefficients of $\mathrm{CeNbO}_{4.08}$ are about one magnitude lower than that of $\mathrm{CeNbO}_{4.25}$ (Fig. 6d). This is ascribed to the larger concentration of the mobile charge carriers in $\mathrm{CeNbO}_{4.25}$. However, the oxide ions in $\mathrm{CeNbO}_{4.33}$ phase with more excess oxygen are essentially immobile at the same simulation temperatures compared with $\mathrm{CeNbO}_{4.08}$ and $\mathrm{CeNbO}_{4.25}$. The well-organized mixed corner/edge-sharing zigzag-shaped polyhedral $\left[\mathrm{Nb}_{6} \mathrm{O}_{26}\right]$ chains in $\mathrm{CeNbO}_{4.33}$ phase resulting from the ordered distribution of the excess oxide ions have much less flexibility on the rotation and deformation. This rigidity blocks the transfer of the oxide ions between the polyhedral units. Also the high concentration of excess oxide ions in the oxygen sublattice seems to have a significant blocking effect to the oxide ion migration. The oxide ions in $\mathrm{CeNbO}_{4.33}$ phase get mobile only when the temperature is increased to above $2200^{\circ} \mathrm{C}$ during the MD simulations (Supplementary Fig. 21). Even in that case, the migration path is confined only along the $\left[\mathrm{Nb}_{6} \mathrm{O}_{26}\right]$ chain (Supplementary Fig. 22). The large $E_{\mathrm{a}}(\sim 2.07 \mathrm{eV})$ calculated from the Arrhenius plot of oxygen diffusion coefficients confirm that the oxide ions are hardly mobile in $\mathrm{CeNbO}_{4.33}$ phase (Fig. 6d).

The MD simulations show an increase of $E_{\mathrm{a}}$ for the oxide ion migration with the temperature for both $\mathrm{CeNbO}_{4.08}$ and $\mathrm{CeNbO}_{4.25}$ phases (Fig. 6d), in accordance with the results by Pramana et al. ${ }^{17}$. The low-temperature $E_{\mathrm{a}}$ values $(0.27-0.41 \mathrm{eV})$ are close to those from the conductivity data of $\mathrm{CeNbO}_{4+\delta}(\delta=$ $0.08,0.25,0.33$; Supplementary Fig. 23), which did not show apparent $E_{\mathrm{a}}$ changes when placed under $\mathrm{N}_{2}$ flow and below $600{ }^{\circ} \mathrm{C}$ to maintain their oxygen contents (Supplementary Fig. 2). However, $\mathrm{CeNbO}_{4+\delta}$ displays mixed electronic and oxide ion conduction and it is hard to quantify the electronic and ionic contributions to the conductivity. Therefore, the calculated $E_{\mathrm{a}}$ from the MD simulations at low temperatures here cannot be compared directly with the experimental values from these conductivity measurements. Owing to the instability of the $\mathrm{CeNbO}_{4+\delta}$ phases to lose the extra oxygen at temperatures above $600{ }^{\circ} \mathrm{C}$ even in the $\mathrm{O}_{2}$-rich atmosphere ${ }^{18,20}$, there is no accurate $E_{\mathrm{a}}$ in higher temperature region for direct comparison with the calculated high-temperature $E_{\mathrm{a}}$ value for each phase. However, the high-temperature $E_{\mathrm{a}}$ values for $\mathrm{CeNbO}_{4.08}(0.84 \mathrm{eV})$ and $\mathrm{CeNbO}_{4.25}(0.93 \mathrm{eV})$ are close to the experimental $E_{\mathrm{a}}(\sim 0.99 \mathrm{eV})$ for oxide diffusion in $\mathrm{CeNbO}_{4+\delta}$ from the ${ }^{18} \mathrm{O}$ tracer diffusion measurements by Packer et al. ${ }^{22}$, further validating our MD simulations. The increase of $E_{\mathrm{a}}$ with the temperature may be explained by the fact that the concentration of mobile oxide anions in $\mathrm{CeNbO}_{4+\delta}$ could depend on the temperature. At low temperatures, the loosely bonded oxide ion could be the major mobile oxide ion, while at higher temperatures the more strongly bonded oxide ions start to move and contribute to the oxide ion migration, which requires extra energy and therefore increases the total $E_{\mathrm{a}}$.

In nonstoichiometric $\mathrm{CeNbO}_{4+\delta}$, the incorporation of extra oxygen atoms is coupled by the oxidation of $\mathrm{Ce}^{3+}$ to smaller $\mathrm{Ce}^{4+}$ and the interstitial oxide ion migrations in the scheelite-based $\mathrm{CeNbO}_{4.08}$ and $\mathrm{CeNbO}_{4.25}$ take place through a synergic mechanism of breaking and reformation of the $\mathrm{Nb}_{2} \mathrm{O}_{9}$ dimers, which is possible thanks to the spatial proximity of the tetrahedral units. This is akin to the continuous breaking and reformation of the tetrahedral dimers in the vacancy-mediated oxide ion conductors based on isolated tetrahedral anion structures, e.g., $\mathrm{La}_{1-x} \mathrm{Ba}_{1+x} \mathrm{GaO}_{4-0.5 x}{ }^{31}$ and $\mathrm{Bi}_{1-x} \mathrm{Sr}_{\mathrm{x}} \mathrm{VO}_{4-0.5 x}{ }^{14}$. However, in these two oxygen-vacancy conducting materials, the tetrahedral-dimer-assisted oxide ion migration does not modify the coordination number, while in the case of $\mathrm{CeNbO}_{4+\delta}$ the coordination number in the polyhedral units involved in the oxide ion migration changes. The oxide ion migration mechanism in $\mathrm{CeNbO}_{4+\delta}$ revealed here thus emphasizes the key roles of the coordination-number-variable cations as well as rotation and deformation flexibility of 4/5coordinated polyhedral units for the oxide ion migration. This is generally consistent with the previous findings in interstitial oxide ion conducting apatite $\mathrm{e}^{31}$ and melilite-type materials ${ }^{10}$. Therefore, the structures containing cations forming polyhedral units with rotation, deformation, and coordination flexibility, e.g., Ga, Ti, V, Nb, Mo, and $\mathrm{W}$, are promising candidates for new oxide ion conductors if, at the same time, flexible oxidation state or donor substitution with smaller cations allows the introduction of excess oxide ions.

\section{Discussion}

In summary, atomic structures of three oxygen hyperstoichiometric materials $\left(\mathrm{CeNbO}_{4.08}, \mathrm{CeNbO}_{4.25}, \mathrm{CeNbO}_{4.33}\right)$ were determined by combining data from $3 \mathrm{D}$ ED, SPD, and NPD. The superstructure of $\mathrm{CeNbO}_{4.33}$ and the $(3+1) \mathrm{D}$ incommensurately modulated structure of $\mathrm{CeNbO}_{4.08}$ were obtained for the first time, to the best of our knowledge. The interstitial sites $\mathrm{O}_{i}$ were identified in all the three compounds and the structure analysis elucidates how the structure adapts for the oxygen hyperstoichiometry change, advancing our understanding of the complex $\mathrm{CeNbO}_{4+\delta}$ system. Cationic size contraction of $\mathrm{Ce}$ upon the oxidation allows not only the incorporation of excess oxygen into the host lattice of $\mathrm{CeNbO}_{4}$ but also the relaxation of the $\mathrm{NbO}_{n}$ polyhedra and their interconnection through mixed corner/edgesharing in three dimensions. MD simulations show that, with the inclusion of the excess oxygen into the host lattice of $\mathrm{CeNbO}_{4}$, the oxide ions become mobile in $\mathrm{CeNbO}_{4.08}$ and $\mathrm{CeNbO}_{4.25}$ with coordination-number-variable network but hardly migrate in the $\mathrm{CeNbO}_{4.33}$ phase owing to the ordered distribution of the excess oxide ions and the 6 -coordinated $\left[\mathrm{Nb}_{6} \mathrm{O}_{26}\right]$ polyhedral chain network with constrained deformation and rotation. Two kinds of oxide ion migration events are identified in $\mathrm{CeNbO}_{4.08}$ and $\mathrm{CeNbO}_{4.25}$ involving the interstitial $\mathrm{O}_{\mathrm{i}}$ sites: (i) migration between the $\mathrm{NbO}_{n}$ polyhedra isolated by Ce cations; (ii) migration between neighboring $\mathrm{NbO}_{n}$ polyhedra. These two processes together form a long-range $3 \mathrm{D}$ network of migration pathways through which the oxygen ions migrate via a synergiccooperation knock-on mechanism involving the continuous breaking and reformation of the $\mathrm{Nb}_{2} \mathrm{O}_{9}$ units assisted by the polyhedral rotation and deformation. The relationship between the structure and oxide ion migration for the whole series of $\mathrm{CeNbO}_{4+\delta}$ compounds here provides means to optimize the performance of these compounds and to develop better oxygen hyperstoichiometric materials for a wide variety of applications.

\section{Methods}

Materials. The parent material $\mathrm{CeNbO}_{4}$ was prepared by traditional solid-state reaction. The powders of $\mathrm{CeO}_{2}$ and $\mathrm{Nb}_{2} \mathrm{O}_{5}$ were mixed and homogenized through grinding with an agate mortar and a pestle. The mixtures were annealed at $1623 \mathrm{~K}$ for $10 \mathrm{~h}$ and then sintered at $1073 \mathrm{~K}$ for $10 \mathrm{~h}$ in flowing argon gas atmosphere. The yellow colored powder, pure phase of $\mathrm{CeNbO}_{4}$, was finally obtained. The oxidized 
phases $\mathrm{CeNbO}_{4.08}, \mathrm{CeNbO}_{4.25}$, and $\mathrm{CeNbO}_{4.33}$ were synthesized as described in Supplementary Fig. 1. In short, the $\mathrm{CeNbO}_{408}$ phase was obtained by heating $\mathrm{CeNbO}_{4}$ in air at $1123 \mathrm{~K}$ for $15 \mathrm{~min}$, then quenching in the furnace to $948 \mathrm{~K}$, holding at $948 \mathrm{~K}$ for another $20 \mathrm{~min}$, and finally quenching to room temperature in air. $\mathrm{CeNbO}_{4.25}$ was prepared by heating $\mathrm{CeNbO}_{4}$ at $873 \mathrm{~K}$ in air for $24 \mathrm{~h}$ and then cooling in furnace to room temperature. The $\mathrm{CeNbO}_{4.33}$ was prepared by heating $\mathrm{CeNbO}_{4}$ at $673 \mathrm{~K}$ in flowing oxygen atmosphere for $72 \mathrm{~h}$ and then cooling to room temperature under oxygen atmosphere. The powder was reground and then reheated under the same conditions until the powder was in a single crystalline phase (Schematic representation of the synthesis of $\mathrm{CeNbO}_{4+\delta}$ is shown in Supplementary Fig. 1).

\section{Characterizations. 3D ED data was collected on a $200 \mathrm{kV}$ JEOL JEM-2100} transmission electron microscope. The goniometer was continuously rotated while SAED patterns were simultaneously captured from crystals with the sizes ranging from 100 to $500 \mathrm{~nm}$ using the quad hybrid pixel detector (Timepix). The 3D ED datasets were then processed using the X-ray Detector Software package ${ }^{32}$, which can export the results as an hkl list. TGA was performed on a TGA-Q500 from room temperature to $1000^{\circ} \mathrm{C}$ with a heating rate of $10^{\circ} \mathrm{C} \mathrm{min}-1$ under flowing $\mathrm{N}_{2}$ atmosphere. The AC impedance spectroscopy (IS) measurements were performed with a Solartron 1260 frequency response analyzer over the $10^{-1}-10^{-7} \mathrm{~Hz}$ frequency range within the $100-700^{\circ} \mathrm{C}$ temperature range under $\mathrm{N}_{2}$ atmosphere flows. Prior to the IS measurements, the platinum paste was coated on the opposite faces of the pellets to form electrodes. Conventional PXRD pattern for parent phase $\mathrm{CeNbO}_{4}$ was collected at room temperature on a PANalytical X'Pert Pro diffractometer in Debye-Scherrer geometry with $\mathrm{Cu} \mathrm{Kal}$ radiation with a minimum full width half maximum of $0.028^{\circ}$. NPD data were collected on $\mathrm{CeNbO}_{4+\delta}$ at ambient temperature over the $10-120^{\circ} 2 \theta$ range at $2 \theta$ intervals of $0.05^{\circ}$ on the $3 \mathrm{~T} 2$ diffractometer at Laboratoire Leon Brillouin (France) using wavelength $\lambda=$ $1.22997 \AA\left(\mathrm{CeNbO}_{4.08}\right.$ and $\left.\mathrm{CeNbO}_{4.33}\right)$ and $\lambda=1.54 \AA\left(\mathrm{CeNbO}_{4.25}\right)$. High-intensity and high-resolution SPD data were recorded on $\mathrm{CeNbO}_{4+\delta}(\delta=0.08,0.25$ and 0.33 ) on the 11BM diffractometer at the Advanced Photon Source, Argonne National Laboratory. SPD data were collected over the $0.5-40^{\circ} 2 \theta$ range with a $0.001^{\circ}$ step size at room temperature with $0.3 \mathrm{~mm}$ sample capillary using $\lambda=$ $0.4130370 \AA$ A. The structure models derived from the 3D ED data were used as initial models for Rietveld refinements by combining SPD with NPD using Jana $2006^{33}$ for $(3+1) \mathrm{D}$ incommensurately modulated structure of $\mathrm{CeNbO}_{4.08}$ and the software Topas Academic Version $5^{34}$ for $\mathrm{CeNbO}_{4.25}$ and $\mathrm{CeNbO}_{4.33}$.

MD simulations. The oxide ion migration in $\mathrm{CeNbO}_{4+\delta}$ was investigated through MD atomistic simulations based on interatomic potential approach with the DL_POLY code ${ }^{35,36}$. The Buckingham potential function ${ }^{37}$ was used to model interactions between ions and the shell model ${ }^{38}$ to describe the electronic polarizability. The interatomic potential parameters, which were used in the previous atomistic simulations of $\mathrm{CeNbO}_{4}$ and $\mathrm{CeNbO}_{4.25}$ by Pramana et al. ${ }^{17}$, were slightly modified (Supplementary Table 4), especially regarding the $\mathrm{Ce}^{4+}-\mathrm{O}^{2-}$ potential parameters ${ }^{39}$ for better reproduction of all experimental $\mathrm{CeNbO}_{4+\delta}$ structures (Supplementary Table 5) by the General Utility Lattice Program (GULP) 39,40 . The lattice parameters and most of the bond lengths were reproduced within $\pm 6 \%$ error except for 2-4 bond lengths in each oxidized phase showing relatively large discrepancies $( \pm 10-20 \%)$ from the experimental values. The MD simulations were performed for the whole series of $\mathrm{CeNbO}_{4+\delta}$ using the updated interatomic potential parameters. The simulation box consisted of a $6 \times 3 \times 6$ supercell containing 2592 atoms for the parent $\mathrm{CeNbO}_{4}$, a $1 \times 3 \times 1$ supercell containing 2112 atoms for $\mathrm{CeNbO}_{4.08}$, a $3 \times 2 \times 4$ supercell containing 7200 atoms for the $\mathrm{CeNbO}_{4,25}$, and a $5 \times 5 \times 3$ supercell containing 2850 atoms for $\mathrm{CeNbO}_{4.33}$. The systems were equilibrated first under a constant pressure of $1 \mathrm{~atm}$ at specific temperatures within $1273-2673 \mathrm{~K}$ for $10^{5}$ time steps with a time step of $0.1 \mathrm{fs}$ before carrying out the main MD simulation for 200 ps with $2 \times 10^{6}$ time steps in the NVT ensemble. The Visual Molecular Dynamics package ${ }^{41}$ was used to perform MD data analysis and the MSDs were calculated with the nMoldyn 3 code ${ }^{42}$. Oxygen diffusion coefficients were calculated from the slope of the MSD plots as a function of simulation time.

\section{Data availability}

All relevant data supporting the findings of this study are available from the corresponding authors upon request.

Received: 22 December 2019; Accepted: 25 August 2020; Published online: 21 September 2020

\section{References}

1. Forslund, R. P. et al. Exceptional electrocatalytic oxygen evolution via tunable charge transfer interactions in $\mathrm{La}_{0.5} \mathrm{Sr}_{1.5} \mathrm{Ni}_{1-\mathrm{x}} \mathrm{Fe}_{\mathrm{x}} \mathrm{O}_{4} \pm \delta$ Ruddlesden-Popper oxides. Nat. Commun. 9, 3150 (2018).
2. Halat, D. M. et al. Probing oxide-ion mobility in the mixed ionic-electronic conductor $\mathrm{La}_{2} \mathrm{NiO}_{4+\delta}$ by solid-state ${ }^{17} \mathrm{O}$ MAS NMR spectroscopy. J. Am. Chem. Soc. 138, 11958-11969 (2016).

3. Nicoud, $\mathrm{S}$. et al. Comprehensive study of oxygen storage in $\mathrm{YbFe}_{2} \mathrm{O}_{4+\mathrm{x}}(\mathrm{x} \leq$ 0.5): unprecedented coexistence of $\mathrm{FeO}_{\mathrm{n}}$ polyhedra in one single phase. J. Am. Chem. Soc. 139, 17031-17043 (2017).

4. Belik, A. A. et al. Crystal and magnetic structures and properties of $\mathrm{BiMnO}_{3+\delta}$. J. Am. Chem. Soc. 132, 8137-8144 (2010).

5. Saranya, A. M. et al. Engineering mixed ionic electronic conduction in $\mathrm{La}_{0.8} \mathrm{Sr}_{0.2} \mathrm{MnO}_{3+\delta}$ nanostructures through fast grain boundary oxygen diffusivity. Adv. Energy Mater. 5, 1500377 (2015).

6. Phillips, J. C. et al. Interstitial oxygen and high-temperature superconductivity in $\mathrm{La}_{2-\mathrm{x}} \mathrm{Sr}_{\mathrm{x}} \mathrm{CuO}_{4+\delta}$. Phys. Rev. B 42, 6795 (1990).

7. Chmaissem, O., Zheng, H., Huq, A., Stephens, P. W. \& Mitchell, J. F. Formation of $\mathrm{Co}^{3+}$ octahedra and tetrahedra in $\mathrm{YBaCo}_{4} \mathrm{O}_{8.1}$. J. Solid State Chem. 181, 664-672 (2008).

8. Huq, A. et al. Structural and magnetic properties of the Kagomé antiferromagnet $\mathrm{YbBaCo}_{4} \mathrm{O}_{7}$. J. Solid State Chem. 179, 1136-1145 (2006).

9. Nakayama, S., Kageyama, T., Aono, H. \& Sadaoka, Y. Ionic-conductivity of lanthanoid silicates, $\mathrm{Ln}_{10}\left(\mathrm{SiO}_{4}\right)_{6} \mathrm{O}_{3}(\mathrm{Ln}=\mathrm{La}, \mathrm{Nd}, \mathrm{Sm}, \mathrm{Gd}, \mathrm{Dy}, \mathrm{Y}, \mathrm{Ho}, \mathrm{Er}$ and Yb). J. Mater. Chem. 5, 1801-1805 (1995).

10. Kuang, X. et al. Interstitial oxide ion conductivity in the layered tetrahedral network melilite structure. Nat. Mater. 7, 498-504 (2008).

11. Boehm, E. et al. Oxygen transport properties of $\mathrm{La}_{2} \mathrm{Ni}_{1-\mathrm{x}} \mathrm{Cu}_{\mathrm{x}} \mathrm{O}_{4+\delta}$ mixed conducting oxides. Solid State Sci. 5, 973-981 (2003).

12. Allen, G. C., Tempest, P. A. \& Tyler, J. W. Coordination model for the defect structure of hyperstoichiometric $\mathrm{UO}_{2+\mathrm{x}}$ and $\mathrm{U}_{4} \mathrm{O}_{9}$. Nature 295, 48-49 (1982).

13. Wang, J. et al. Molecular dynamic simulation of interstitial oxide ion migration in $\mathrm{Pb}_{1-\mathrm{x}} \mathrm{La}_{\mathrm{x}} \mathrm{WO}_{4+\mathrm{x} / 2}$ scheelite. J. Solid State Chem. 268, 16-21 (2018).

14. Yang, X. Y. et al. Cooperative mechanisms of oxygen vacancy stabilization and migration in the isolated tetrahedral anion scheelite structure. Nat. Commun. 9, 4484 (2018).

15. Cava, J. R. \& Roth, R. S. Characterisation of modulated structures in $\mathrm{ABO}_{4+\mathrm{x}}$ features. AIP Conf. Proc. 53, 361 (1979).

16. Thompson, J. G., Withers, R. L. \& Brink, F. J. Modulated structures in oxidized cerium niobates. J. Solid State Chem. 143, 122-131 (1999).

17. Pramana, S. S. et al. Correlation of local structure and diffusion pathways in the modulated anisotropic oxide ion conductor $\mathrm{CeNbO}_{4.25}$. J. Am. Chem. Soc. 138, 1273-1279 (2016).

18. Vullum, F. \& Grande, T. Oxidation driven decomposition of $\mathrm{CeNbO}_{4}$ in pure oxygen. Chem. Mater. 20, 5434-5437 (2008).

19. Vullum, F. \& Grande, T. Oxygen stoichiometry and transport properties of cerium niobite. Solid State Ion. 179, 1061-1065 (2008).

20. Skinner, S. J. \& Kang, Y. X-ray diffraction studies and phase transformations of $\mathrm{CeNbO}_{4+\delta}$ using in situ techniques. Solid State Sci. 5, 1475-1479 (2003).

21. Skinner, S. J. et al. Redox chemistry of the novel fast oxide ion conductor $\mathrm{CeNbO}_{4+\mathrm{d}}$ determined through an in-situ spectroscopic technique. Solid State Ion. 192, 659-663 (2011).

22. Packer, R. J. \& Skinner, S. J. Remarkable oxide ion conductivity observed at low temperatures in a complex superstructured oxide. Adv. Mater. 22, 1613-1616 (2010).

23. Bayliss, R. D. Fergusonite-type $\mathrm{CeNbO}_{4+\delta}$ : single crystal growth, symmetry revision and conductivity. J. Solid State Chem. 204, 291-297 (2013).

24. Li, J. \& Sun, J. Application of X-ray diffraction and electron crystallography for solving complex structure problems. Acc. Chem. Res. 50, 2737-2745 (2017).

25. Li, J. et al. Discovery of complex metal oxide materials by rapid phase identification and structure determination. J. Am. Chem. Soc. 141, 4990-4996 (2019).

26. Wan, W., Sun, J., Su, J., Hovmoller, S. \& Zou, X. Three dimensional rotation electron diffraction software RED for automated data collection and data processing. J. Appl. Cryst. 46, 1863-1873 (2013).

27. Yun, Y. F. et al. Phase identification and structure determination from multiphase crystalline powder samples by rotation electron diffraction. J. Appl. Cryst. 47, 2048-2054 (2014).

28. Sheldrick, G. M. SHELXT-Integrated space-group and crystal-structure determination. Acta Cryst. A71, 3-8 (2015).

29. Palatinus, L. \& Chapuis, G. SUPERFLIP- a computer program for the solution of crystal structures by charge flipping in arbitrary dimensions. J. Appl. Crystallogr. 40, 786-790 (2007).

30. $\mathrm{Wu}$, J. et al. Ab initio phasing of X-ray powder diffraction patterns by charge flipping. Nat. Mater. 5, 647-652 (2006).

31. Kendrick, E., Kendrick, J., Knight, K. S., Islam, M. S. \& Slater, P. R. Cooperative mechanisms of fast-ion conduction in gallium-based oxides with tetrahedral moieties. Nat. Mater. 6, 871-875 (2007).

32. Kabsch, W. Integration, scaling, space-group assignment and post-refinement. Acta Cryst. D66, 125-132 (2010). 
33. Petrícek, V., Dusek, M. \& Palatinus, L. Crystallographic computing system JANA2006: general features. Z. Kristallogr. 229, 345-352 (2014).

34. Coelho, A. Topas academic version 5. http://www.topas-academic.net/ (2012).

35. Todorov, I. T., Smith, W., Trachenko, K. \& Dove, M. T. DL_POLY_3: new dimensions in molecular dynamics simulations via massive parallelism. $J$. Mater. Chem. 16, 1911-1918 (2006).

36. Islam, $\mathrm{M}$. $\mathrm{S}$. Ionic transport in $\mathrm{ABO}_{3}$ perovskite oxides: a computer modelling tour. J. Mater. Chem. 10, 1027-1038 (2000).

37. Dick, B. G. Jr. \& Overhauser, A. W. Theory of the dielectric constants of alkali halide crystals. Phys. Rev. 112, 90-103 (1958).

38. Lewis, G. V. \& Catlow, C. R. A. Potential models for ionic oxides. J. Phys. C Solid State Phys. 18, 1149-1161 (1985).

39. Gale, J. D. GULP: a computer program for the symmetry-adapted simulation of solids. J. Chem. Soc. Faraday Trans. 93, 629-637 (1997).

40. Gale, J. D. \& Rohl, A. L. The General Utility Lattice Program (GULP). Mol. Simul. 29, 291-341 (2003).

41. Humphrey, W., Dalke, A. \& Schulten, K. VMD: Visual Molecular Dynamics. J. Mol. Graph. Model 14, 33-38 (1996).

42. Rog, T., Murzyn, K., Hinsen, K. \& Kneller, G. R. nMoldyn: a program package for a neutron scattering oriented analysis of molecular dynamics simulations. J. Comput. Chem. 24, 657-667 (2003).

\section{Acknowledgements}

J.S. gratefully acknowledges financial support from the National Natural Science Foundation of China (21527803, 21471009 and 21621061). X.K. acknowledges financial support from the National Natural Science Foundation of China (21622101 and 21511130134) and Guangxi Natural Science Foundation (2019GXNSFGA245006 and 2014GXNSFGA118004). We also acknowledge the Swedish Research Council (VR), the Knut and Alice Wallenberg Foundation (KAW), and beamline Laboratoire Leon Brillouin (France).

\section{Author contributions}

J. Li performed the SAED data collection, 3D ED data collection, and the structure solution and refinement of $\mathrm{CeNbO}_{4+\delta}$. S.G. and X.K. performed the molecular dynamic simulations. F.P. and C.L. performed the syntheses, PXRD data collection, and assisted with refinement. M.A. performed the SPD and NPD data collection. L.P. supervised the incommensurately modulated structure solution and refinement. J.S. and X.K. conceived the project and supervised the experiments. J.S., X.K., L.P., and J. Li wrote the manuscript with the assistance of the other authors.

\section{Competing interests}

The authors declare no competing interests.

\section{Additional information}

Supplementary information is available for this paper at https://doi.org/10.1038/s41467 020-18481-x.

Correspondence and requests for materials should be addressed to X.K. or J.S.

Peer review information Nature Communications thanks the anonymous reviewers for their contributions to the peer review of this work.

Reprints and permission information is available at http://www.nature.com/reprints

Publisher's note Springer Nature remains neutral with regard to jurisdictional claims in published maps and institutional affiliations.

(C) Open Access This article is licensed under a Creative Commons Attribution 4.0 International License, which permits use, sharing, adaptation, distribution and reproduction in any medium or format, as long as you give appropriate credit to the original author(s) and the source, provide a link to the Creative Commons license, and indicate if changes were made. The images or other third party material in this article are included in the article's Creative Commons license, unless indicated otherwise in a credit line to the material. If material is not included in the article's Creative Commons license and your intended use is not permitted by statutory regulation or exceeds the permitted use, you will need to obtain permission directly from the copyright holder. To view a copy of this license, visit http://creativecommons.org/ licenses/by/4.0\%

(C) The Author(s) 2020 